\title{
Circulating fibrocytes traffic to the lungs in response to CXCL12 and mediate fibrosis
}

\author{
Roderick J. Phillips, ${ }^{1}$ Marie D. Burdick, ${ }^{1}$ Kurt Hong, ${ }^{2}$ Marin A. Lutz, ${ }^{1}$ Lynne A. Murray, ${ }^{1}$ \\ Ying Ying Xue,1 John A. Belperio, ${ }^{1}$ Michael P. Keane,1 and Robert M. Strieter ${ }^{1,3}$
}

1Division of Pulmonary and Critical Care Medicine, 2Division of Nutrition, and ${ }^{3}$ Departments of Pathology and Pediatrics, David Geffen School of Medicine at University of California - Los Angeles, Los Angeles, California, USA.

\begin{abstract}
Previous reports have identified a circulating pool of $\mathrm{CD}^{4} 5^{+}$collagen $\mathrm{I}^{+} \mathrm{CXCR}^{+}\left(\mathrm{CD} 45^{+} \mathrm{Col} \mathrm{I}{ }^{+} \mathrm{CXCR} 4^{+}\right)$cells, termed fibrocytes, that traffic to areas of fibrosis. No studies have demonstrated that these cells actually contribute to fibrosis, however. Pulmonary fibrosis was originally thought to be mediated solely by resident lung fibroblasts. Here we show that a population of human $\mathrm{CD}^{+} 5^{+} \mathrm{Col} \mathrm{I}^{+} \mathrm{CXCR} 4^{+}$circulating fibrocytes migrates in response to CXCL12 and traffics to the lungs in a murine model of bleomycin-induced pulmonary fibrosis. Next, we demonstrated that murine $\mathrm{CD}^{+} 5^{+} \mathrm{Col} \mathrm{I}^{+} \mathrm{CXCR} 4^{+}$fibrocytes also traffic to the lungs in response to a bleomycin challenge. Maximal intrapulmonary recruitment of $\mathrm{CD} 45^{+} \mathrm{Col} \mathrm{I}^{+} \mathrm{CXCR} 4^{+}$fibrocytes directly correlated with increased collagen deposition in the lungs. Treatment of bleomycin-exposed animals with specific neutralizing anti-CXCL12 Ab's inhibited intrapulmonary recruitment of CD $45^{+} \mathrm{Col} \mathrm{I}^{+} \mathrm{CXCR} 4^{+}$circulating fibrocytes and attenuated lung fibrosis. Thus, our results demonstrate, we believe for the first time, that circulating fibrocytes contribute to the pathogenesis of pulmonary fibrosis.
\end{abstract}

\section{Introduction}

Idiopathic pulmonary fibrosis (IPF) is a chronic and often fatal pulmonary disorder with a survival rate as low as $30 \% 5$ years after diagnosis $(1,2)$. The prevalence of this disease increases with age - with an incidence as high as 250 cases per 100,000 in the elderly $(2,3)$. There also appears to be an increase in the incidence of the disease in many Western countries, including the United Kingdom, New Zealand, and Germany (3). Conventional approaches to treating IPF have focused on immunosuppressive therapy, predominantly steroid usage, which has yielded poor results. The pathogenesis of pulmonary fibrosis is characterized by dysregulated and inappropriate tissue remodeling and enhanced vascular remodeling, fibroproliferation, and deposition of ECM (4). Without resolution, continued fibrosis leads to loss of lung function and ultimately death.

Fibrocytes present in the peripheral circulation were first identified a decade ago (5). These cells comprised a minor component of the circulating pool of leukocytes (less than 1\%) and express a characteristic pattern of markers, including collagen I (Col I) and CD45 (5). When cultured in vitro, these cells became adherent and developed a spindle-shaped morphology (5). Subsequent studies have revealed that these circulating fibrocytes express chemokine receptors such as CXCR4 and CCR7 (6). Furthermore, circulating fibrocytes have been shown to traffic to wounds (6). They express ECM proteins, such as pro-Col I and pro-Coll III, which may be involved in connective tissue formation (6-8). No study has actually demonstrated that fibrocytes contribute to deposition of ECM and the promotion of fibrosis in vivo, however.

Tissue fibroblasts have classically been held to be responsible for repair and remodeling within the lung, although this view has

Nonstandard abbreviations used: bronchoalveolar lavage fluid (BAL); collagen I (Col I); idiopathic pulmonary fibrosis (IPF); phycoerythrin (PE); $\alpha$-smooth muscle actin ( $\alpha$-SMA).

Conflict of interest: The authors have declared that no conflict of interest exists.

Citation for this article: J. Clin. Invest. 114:438-446 (2004).

doi:10.1172/JCI200420997. been challenged since those fibroblasts observed in the fibrotic lung show phenotypic differences to their counterparts in the lungs of healthy individuals $(9,10)$. Thus, it is possible that, in common with their perceived role in fibrosis and wound repair, circulating fibrocytes may also be recruited to injured lungs as an integral component of the pathogenesis of pulmonary fibrosis.

\section{Results}

Human fibrocytes express $C D 45$, Col I, and CXCR4 and migrate in response to CXCL12. To dissect the role of fibrocytes in pulmonary fibrosis, we initially isolated and characterized a population of fibrocytes from human peripheral blood (Figure 1 and Table 1). Although these cells are a minor component of the PBMC pool, by using a technique of immunodepletion and culturing cells in vitro, we were able to purify a population of long, spindle-shaped cells that morphologically resemble fibrocytes (6). We began our characterization of these purified fibrocytes by using flow cytometry to assess changes in the expression of the key fibrocyte markers CD45, CD34, Col I, and $\alpha$-smooth muscle actin ( $\alpha$-SMA) as the cells matured in culture (Table 1). Our results show that these newly purified fibrocytes expressed high levels of CD45, CD34, and Col I, yet after 1 week in culture, expression of CD34 levels dropped dramatically and continued to decrease throughout the duration of the experiment (Table 1). CD45 expression began to fall at week 2 , and by the third week (28-35 days after cell isolation) there was an approximate threefold reduction in the expression of CD45 by fibrocytes (Table 1). By contrast, Col I expression remained elevated at all time points examined (Table 1). Consistent with other published observations, there was no expression of $\alpha$-SMA in freshly purified fibrocytes (6, 11 ), but after 3 weeks in culture almost one-third of fibrocytes were found to stain positive for $\alpha$-SMA (Table 1 ). Furthermore, addition of TGF- $\beta 1$ to cultured fibrocytes enhanced the kinetics of this differentiation (data not shown), which is again consistent with published observations $(6,11)$. Changes in the cell surface phenotype of cultured fibrocytes similar to those described above were also observed by immunostaining (Figure 1A). Here, purified fibrocytes 

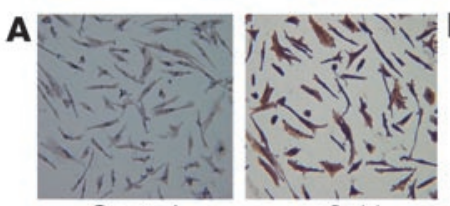

Control

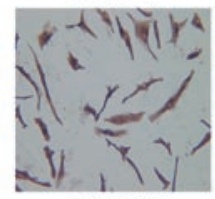

CXCR4

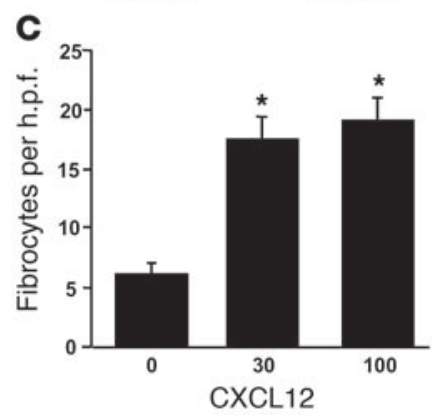

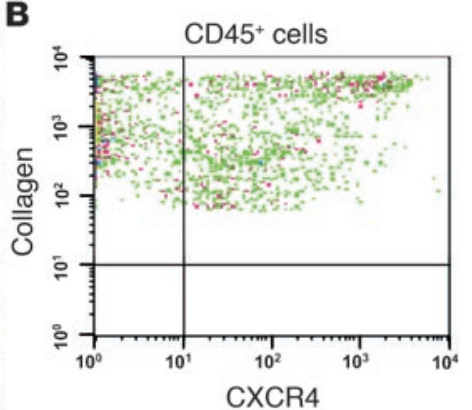

D

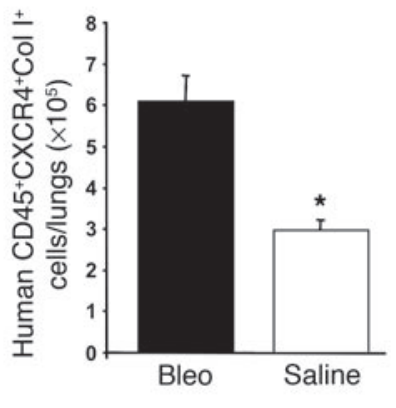

\section{Figure 1}

Characterization of human fibrocytes and their trafficking to bleomycin-induced lung fibrosis in SCID mice. (A) Human fibrocytes stained with control $\mathrm{Ab}$, human $\mathrm{Col} \mathrm{I}$, human $\mathrm{CXCR} 4$, and human $\alpha$-SMA 3 weeks after purification. Representative fields viewed at $\times 400$ magnification. (B) Isolated human fibrocytes triple-stained for Col I, CD45, and CXCR4 were examined by FACS analysis. Data shows CD45+ fibrocytes that were examined for dual expression of CXCR4 and Col I. (C) Isolated human fibrocyte chemotaxis in response to 0,30 , and $100 \mathrm{ng} / \mathrm{ml}$ of CXCL12. Data are representative of three experiments. ${ }^{*} P<0.05$. h.p.f., high-powered field. (D) Isolated human fibrocytes $\left(10^{6}\right)$ were injected into the tail vein of SCID mice at day 4 after treatment with either intratracheal bleomycin (Bleo) or saline, followed by recovery of cells from the lungs at day 8. Cells were then stained for human CD45, Col I, and CXCR4 and analyzed by flow cytometry to determine trafficking of human fibrocytes to the lungs of SCID mice. ${ }^{*} P<0.05$. cultured for 3 weeks (28-35 days after their initial isolation) were found to express intracellular Col I (Figure 1A), cell surface CXCR4 (Figure $1 \mathrm{~A}$ ), and $\alpha$-SMA (Figure $1 \mathrm{~A}$ ).

Next, we triple-stained newly purified fibrocytes with Col I, CD45, and CXCR4 and analyzed them by flow cytometry (Figure 1B). Initially, we gated $\mathrm{CD} 45^{+}$fibrocytes and then examined the resulting population for dual expression of CXCR4 and Col I. In this way, we identified a major population of CD $45^{+} \mathrm{Col} \mathrm{I}{ }^{+} \mathrm{CXCR} 4^{+}$ human fibrocytes and a smaller population of $\mathrm{CD} 45^{+} \mathrm{Col} \mathrm{I} \mathrm{I}^{+} \mathrm{CXCR} 4^{-}$ fibrocytes (Figure 1B). To determine whether the expression of CXCR4 on the surface of purified fibrocytes was functional, we performed chemotaxis assays to look for specific migration in response to CXCL12. We observed significant chemotaxis at both 30 and $100 \mathrm{ng} / \mathrm{ml} \mathrm{CXCL12} \mathrm{(Figure} \mathrm{1C),} \mathrm{indicating} \mathrm{that}$ $\mathrm{CD} 45^{+} \mathrm{Col} \mathrm{I}{ }^{+} \mathrm{CXCR} 4^{+}$fibrocytes undergo directed movement in the presence of a chemotactic gradient in vitro.

Having established that cultured human fibrocytes can migrate in response to CXCL12 in vitro, we wanted to examine whether these fibrocytes could migrate in vivo and, in particular, whether they could specifically migrate into lungs undergoing a fibrotic response. To test this possibility we used a murine model of bleomycin-induced pulmonary fibrosis. Here, we performed tailvein injections of purified human fibrocytes into SCID mice that had already been exposed to either bleomycin or saline for 4 days. After a further 4 days (i.e., 8 days after the initial instillation of either bleomycin or saline), the mice were sacrificed and the lungs analyzed for the presence of infiltrating human fibrocytes. Our results (Figure 1D) demonstrated significantly greater numbers of human $\mathrm{CD}_{4} 5^{+} \mathrm{Col} \mathrm{I} \mathrm{I}^{+} \mathrm{CXCR} 4^{+}$fibrocytes were observed in bleomycin-treated lungs compared with those lungs that received saline alone. These results suggest that a circulating pool of human fibrocytes exist that specifically migrate in response to CXCR4/ CXCL12 and traffic to fibrotic lungs.

A kinetic analysis of the onset of fibrosis reveals dramatic collagen deposition beginning 4 days after bleomycin exposure. To more fully elucidate the role of fibrocytes in the development of fibroproliferative disorders of the lung, we focused our studies on the murine model of bleomycin-induced pulmonary fibrosis (12). Initially, cohorts of mice were treated with either bleomycin, saline, or nothing (naive mouse, day 0) and then sacrificed at day 1, 2, 4, 8, 16, and 20 to look for evidence of fibrosis. Exuberant collagen deposition within the lung is a key marker of fibrosis (9); on this basis we examined changes in pro-Col I and pro-Col III gene expression using realtime quantitative RT-PCR (Figure 2, A and B). Our data revealed that transcription of both pro-Col III and pro-Col I was dramatically upregulated in mice exposed to bleomycin (up to 30-fold higher) as compared with mice treated with saline alone (Figure 2, A and B). To confirm that collagen gene expression was translated into protein, we next measured total collagen deposition in the lung by the Sircol assay (13). We observed that the level of total

\section{Table 1}

Kinetics of percentage of positive Col I, CD45, CD34, and $\alpha$-SMA expression in isolated and purified human fibrocytes as determined by FACS

$\begin{array}{lcc}\text { Marker } & \begin{array}{c}\mathbf{7 - 1 4} \mathbf{d} \text { after cell isolation } \\ \text { immediately after purification }\end{array} & \begin{array}{c}\mathbf{1 4 - 2 1} \mathbf{d} \text { after cell isolation } \\ \mathbf{1} \text { wk after purification }\end{array} \\ \text { Col I } & 90 & 90 \\ \text { CD45 } & 90 & 85 \\ \text { CD34 } & 90 & 18 \\ \alpha \text {-SMA } & 0 & 0\end{array}$

$\mathbf{2 1 - 2 8} \mathbf{d}$ after cell isolation
$\mathbf{2}$ wk after purification
88
54
11
10

28-35 $\mathrm{d}$ after cell isolation 3 wk after purification 91 30

5

30 

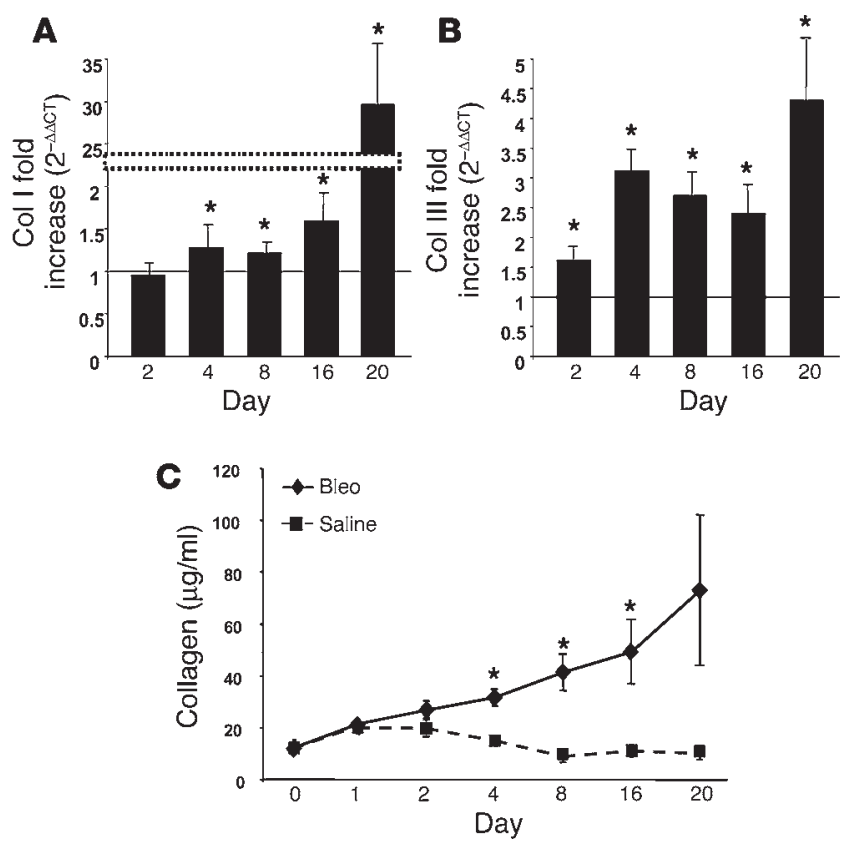

collagen protein increased dramatically in bleomycin-treated mice, especially between days 4 and 20 (Figure 2C). The levels of collagen found in saline-treated mice increased slightly in the first 2 days and then decreased to the levels observed in the naive mouse (day 0 ; Figure 2C). Thus, this data reveals that 4 days after mice have been exposed to bleomycin there is a significant increase in both collagen gene expression and protein deposition, which is indicative of the onset of fibrosis.

$\mathrm{CD} 5^{+} \mathrm{Col} \mathrm{I} \mathrm{I}^{+} \mathrm{CXCR} 4^{+}$fibrocytes accumulate in the lungs of bleomycin-treated animals. To characterize the role of murine $\mathrm{CD} 45^{+} \mathrm{Col} \mathrm{I}{ }^{+} \mathrm{CXCR} 4^{+}$ fibrocytes in our mouse model of bleomycin-induced pulmonary fibrosis, we isolated cells from the lungs and blood buffy coats of mice that had been exposed to bleomycin or saline for $1,2,4,8,16$, and 20 days, triple-stained for CD45, Col I, and CXCR4, and examined them by flow cytometry (Figure $3 \mathrm{~A}$ ). Our results reveal a dramatic increase in the number of infiltrating $\mathrm{CD} 45^{+} \mathrm{Col} \mathrm{I}{ }^{+} \mathrm{CXCR} 4^{+}$ fibrocytes found in the lungs of mice exposed to bleomycin. Increased expression of $\mathrm{CD}_{4} 5^{+} \mathrm{Col} \mathrm{I} \mathrm{I}^{+} \mathrm{CXCR} 4^{+}$cells began to appear 2 days after bleomycin treatment, became maximal at day 8 , and remained elevated at days 16 and 20 . Expression of $\mathrm{CD} 45^{+} \mathrm{Col} \mathrm{I} \mathrm{I}^{+} \mathrm{CXCR} 4^{+}$cells in saline-treated mice also increased initially before returning to the levels observed in the naive mouse (day 0) by days 16 and 20 (Figure $3 \mathrm{~A}$ ). The most likely explanation for this latter phenomenon is that intratracheal instillation of saline itself is not a normal event and could have promoted an inflammatory response. In contrast to the time-dependent accumulation of murine fibrocytes in the lungs of bleomycin-exposed animals, the steady-state levels of circulating $\mathrm{CD} 5^{+} \mathrm{Col} \mathrm{I}{ }^{+} \mathrm{CXCR} 4^{+}$fibrocytes in the blood remained similar in both groups (Figure 3A).

Previous studies have reported that fibrocytes can express CCR7 in addition to CXCR4 (6), and we have now identified a second fibrocyte population that is $\mathrm{CD} 45^{+} \mathrm{Col} \mathrm{I}{ }^{+} \mathrm{CCR} 7^{+}$(Figure $3 \mathrm{~B}$ and data not shown). These CCR7 ${ }^{+}$fibrocytes also traffic to the lungs of bleomycin-treated mice (Figure 3B); however, the absolute number of CCR $7^{+}$fibrocytes found in the fibrotic lung is twofold to threefold lower than the number of $\mathrm{CXCR} 4^{+}$fibrocytes present under similar conditions (i.e., compare A and B in Figure 3). Thus,

\section{Figure 2}

Kinetics of Col I and Col III gene expression and total collagen protein deposition in the lung during bleomycin-induced pulmonary fibrosis. (A and B) Kinetics of pro-Col I (A) and pro-Col III (B) gene expression in lungs of mice exposed to intratracheal bleomycin compared with saline control as determined by real-time quantitative PCR. $n=6$ lungs in each group. Data represent the mean \pm SEM. ${ }^{*} P<0.05$, significant differences between bleomycin and saline groups. ct, threshold cycle number. (C) Kinetics of total collagen protein deposition in lungs of mice exposed to either intratracheal bleomycin, saline, or naive (time 0 ) control as determined by the Sircol assay. $n=6$ lungs in each group. Data represent the mean \pm SEM. ${ }^{*} P<0.05$, significant differences between bleomycin and saline groups.

these data suggest that although two chemotactic chemokine receptors are present on the surface of fibrocytes, CXCR4 appears predominant for the recruitment of fibrocytes to fibrotic lungs.

We have already shown that human $\mathrm{CD} 45^{+} \mathrm{Col} \mathrm{I}^{+} \mathrm{CXCR} 4{ }^{+}$fibrocytes traffic through the bloodstream and are recruited into the lungs of bleomycin-treated animals (Figure 1D), and this suggests that murine $\mathrm{CD} 45^{+} \mathrm{Col} \mathrm{I} \mathrm{I}^{+} \mathrm{CXCR} 4^{+}$fibrocytes may be recruited in a similar manner. There appears to be insufficient peripheral blood fibrocytes present to drive the dramatic intrapulmonary recruitment of progenitor cells (Figure 3A), however. One explanation to account for this disparity is that the circulating pool of fibrocytes is continually replenished from the bone marrow. In support of this notion, recent work has suggested that mesenchymal stem cells originating from the bone marrow traffic to organizing alveolitis in pulmonary irradiated bone marrow chimeric mice (14). To formally test this hypothesis, therefore, bone marrow was recovered from mice treated with bleomycin or saline for 8 days, triple-stained for CD45, Col I, and CXCR4, and analyzed by flow cytometry (Figure 3C). Our results clearly indicate a dramatic increase in the number of $\mathrm{CD} 45^{+} \mathrm{Col} \mathrm{I}^{+} \mathrm{CXCR} 4^{+}$fibrocytes found in the bone marrow of bleomycin-challenged animals in comparison with the normal saline control mice. These data therefore suggest that bone marrow is at least one source of $\mathrm{CD} 45^{+} \mathrm{Col} \mathrm{I}{ }^{+} \mathrm{CXCR} 4^{+}$ circulating fibrocytes and that fibrocyte numbers in the bone marrow appear to be higher in mice treated with bleomycin.

For $\mathrm{CD}_{45} \mathrm{Col} \mathrm{I}^{+} \mathrm{CXCR} 4^{+}$fibrocytes to enter the lungs from the peripheral circulation, the cells must be actively recruited. One likely mechanism for achieving this is the chemokine receptor CXCR4 and its cognate ligand CXCL12, which has been shown to regulate cellular migration in other systems $(6,15,16)$. To establish whether indeed a CXCL12 gradient exists between the lungs and the peripheral blood, lung homogenates were prepared and the plasma isolated from naive, saline-, or bleomycin-treated animals at $1,2,8,16$, and 20 days and the levels of CXCL12 protein determined by ELISA (Figure 3D). Whereas plasma steady-state levels of CXCL12 protein from either bleomycin- or saline-treated mice remained unchanged throughout the time course of the experiment (at $10-15 \mathrm{pg} / \mathrm{ml}$ ), CXCL12 levels in the lungs of animals exposed to bleomycin for 8 days were significantly higher $(790 \pm 80 \mathrm{pg} / \mathrm{ml})$ than the comparable saline control $(273 \pm 70 \mathrm{pg} / \mathrm{ml})$ or the naive control $(190 \pm 25 \mathrm{pg} / \mathrm{ml}$; Figure 3D). Thereafter, CXCL12 levels in the lungs from saline-treated animals gradually decreased, until by day 20 they approached CXCL12 levels observed in the naive mouse. By contrast, CXCL12 levels in bleomycin-challenged lungs remain elevated until day 20. Although CXCL12 protein in the lungs from saline-treated mice are higher than naive lungs during the earlier time points, 

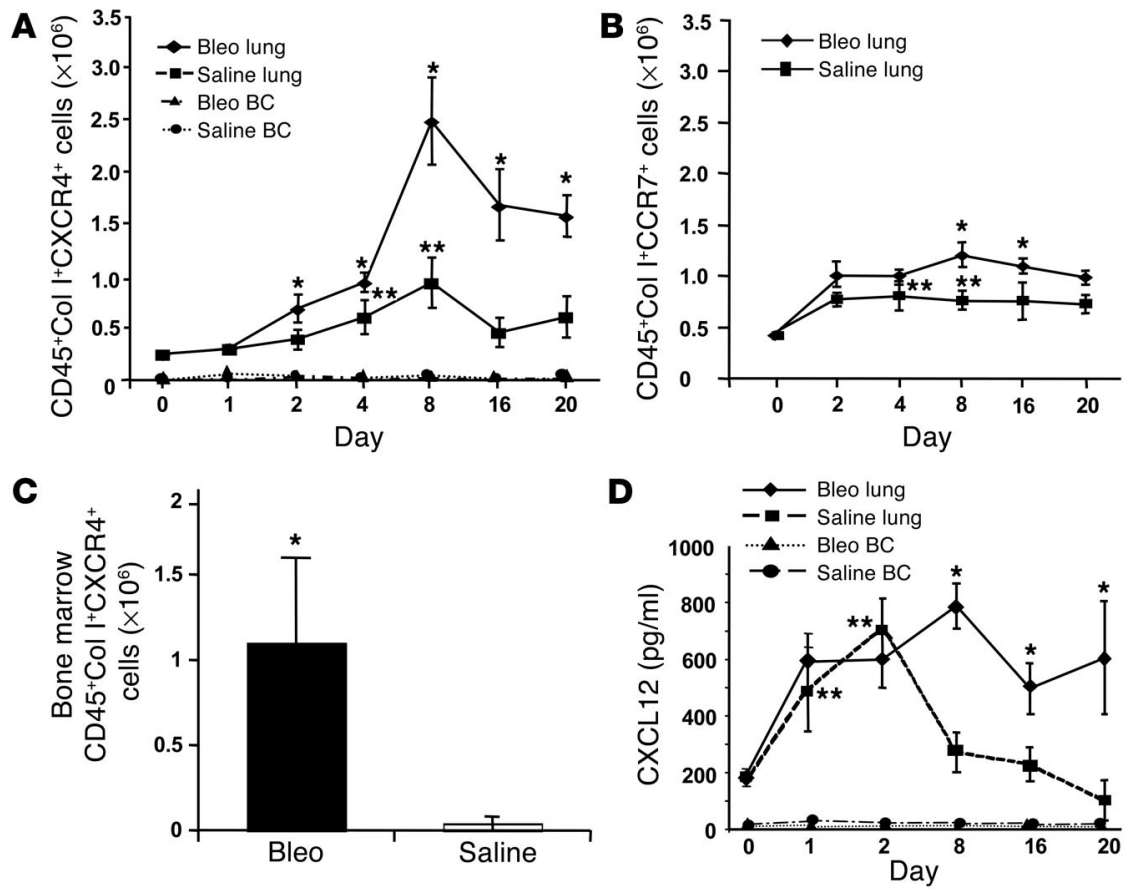

Figure 3

Intrapulmonary recruitment of $\mathrm{CD} 45^{+} \mathrm{Col} \mathrm{I+CXCR} 4{ }^{+}$fibrocytes is greater than $\mathrm{CD} 45^{+} \mathrm{Col} \mathrm{I}^{+}$ CCR7 ${ }^{+}$fibrocytes and correlates with collagen deposition in the lungs of bleomycin-exposed mice. (A and B) Single-cell suspensions were isolated from bleomycin- or saline-challenged lungs and blood buffy coats at the times indicated, triple-stained for CD45, Col I, and CXCR4 (A), or CD45, Col I, and CCR7 (lungs only) (B), and then examined by FACS analysis. $n=6$ samples per group. BC, buffy coat. Data represent the mean \pm SEM. ${ }^{*} P<0.05$, significant differences between bleomycin and saline groups. ${ }^{*} P<0.05$, significant differences between saline-exposed mice and the naive mice. (C) Mice were treated with either intratracheal bleomycin or saline for 8 days. Bone marrow was removed, triple-stained for CD45, Col I, and CXCR4, and then examined by FACS analysis. $n=3$ samples per group. Data represent the mean \pm SEM. ${ }^{*} P<0.05$. (D) Kinetics of CXCL12 protein expression in lung tissue and plasma of mice exposed to either intratracheal bleomycin, saline, or naive control (day 0 ) as determined by ELISA. $n=6$ samples in each group. Data represents the mean \pm SEM. ${ }^{\star} P<0.05$, significant differences between bleomycin and saline groups. ${ }^{* *} P<0.05$, significant differences between saline-exposed mice and the naive mice.

they decreased by day 20, again suggesting that the initial instillation of saline promoted an inflammatory response. Thus, these data support the notion that a CXCL12 gradient exists between the lungs and plasma of bleomycin-treated mice, which could promote the recruitment of $\mathrm{CD}_{4}{ }^{+} \mathrm{Col} \mathrm{I}{ }^{+} \mathrm{CXCR} 4^{+}$fibrocytes to the fibrotic lung. A similar chemokine gradient between the lungs and plasma of bleomycin-treated mice exists for CCL21 (6Ckine), but not for CCL19 (ELC), the putative ligands for CCR7. Here, peak levels of CCL21 in the lung of bleomycin-treated mice occurred at day 8 (402 $\pm 40 \mathrm{pg} / \mathrm{ml})$, while comparable levels of CCL21 in saline-treated mice were $99 \pm 10 \mathrm{pg} / \mathrm{ml}$ at the same time point. Thus CCL21 may promote the recruitment of $\mathrm{CD}_{4}{ }^{+} \mathrm{Col} \mathrm{I}{ }^{+} \mathrm{CCR} 7^{+}$fibrocytes to the fibrotic lung by a mechanism similar to that suggested above for $\mathrm{CD} 45^{+} \mathrm{Col} \mathrm{I}{ }^{+} \mathrm{CXCR} 4^{+}$fibrocytes. It would appear, however, that trafficking of CXCR $4^{+}$fibrocytes predominates over CCR7 ${ }^{+}$fibrocytes during bleomycin-induced pulmonary fibrosis.

Specific neutralizing anti-CXCL12 Ab's block recruitment of $\mathrm{CD} 45^{+} \mathrm{Col} \mathrm{I}^{+}$ CXCR4 $4^{+}$fibrocytes into bleomycin-exposed lungs and attenuates fibrosis. In the light of the results described above, we wanted to determine whether we could specifically block the recruitment of $\mathrm{CD} 45^{+} \mathrm{Col} \mathrm{I}{ }^{+} \mathrm{CXCR} 4{ }^{+}$fibrocytes into the fibrotic lung by inhib- iting the CXCR4/CXCL12 chemotactic axis. To do this we used neutralizing antiCXCL12 Ab's that we have previously shown to specifically and selectively target only CXCL12 (16). Therefore, mice received intratracheal injections of bleomycin together with daily injections of either anti-CXCL12 or control Ab's for 16 days. In addition, one cohort of mice received saline only, and a second cohort received nothing (naive control). Subsequently, all the mice were sacrificed and examined for infiltrating $\mathrm{CD}_{4} 5^{+} \mathrm{Col} \mathrm{I}{ }^{+} \mathrm{CXCR} 4{ }^{+}$fibrocytes by flow cytometry at day 16 (Figure 4A).

Our results show that significantly fewer $\mathrm{CD} 45^{+} \mathrm{Col} \mathrm{I}{ }^{+} \mathrm{CXCR} 4{ }^{+}$fibrocytes $(P<0.05)$ were observed in anti-CXCL12-treated bleomycin-exposed lungs than were observed in lungs exposed to bleomycin in the presence of control Ab's (Figure 4A). Indeed, the number of infiltrating $\mathrm{CD} 45^{+} \mathrm{Col} \mathrm{I}{ }^{+} \mathrm{CXCR} 4^{+}$ fibrocytes observed in the anti-CXCL12treated bleomycin-exposed mice was not significantly different from the number of $\mathrm{CD} 45^{+} \mathrm{Col} \mathrm{I}{ }^{+} \mathrm{CXCR} 4{ }^{+}$fibrocytes found in the lungs of mice exposed to saline alone. And in keeping with our previous observations (Figure 3A), there was a slight increase in the number of $\mathrm{CD}_{4} 5^{+} \mathrm{Col} \mathrm{I}{ }^{+} \mathrm{CXCR} 4^{+}$fibrocytes in the lungs of saline-exposed mice in comparison with the naive control (Figure 4A).

Next we examined the effects of daily injections of anti-CXCL12 Ab's or control Ab's on collagen deposition in the lungs of mice exposed to bleomycin for 16 days (Figure 4B). Our results show that pulmonary collagen deposition was significantly reduced $(P<0.05)$ in anti-CXCL12-treated bleomycin-exposed mice in comparison with those mice receiving bleomycin and control Ab's (Figure 4B). The addition of neutralizing CXCL12 Ab's to bleomycin-treated lungs did not, however, completely attenuate collagen deposition to the level of the saline control (Figure 4B). Taken together, therefore, these data indicate that inhibition of the CXCR4/ CXCL12 chemotactic axis reduces intrapulmonary recruitment of $\mathrm{CD} 5^{+} \mathrm{Col} \mathrm{I}{ }^{+} \mathrm{CXCR} 4^{+}$fibrocytes and significantly abrogates lung fibrosis in bleomycin-exposed mice.

To ascertain whether the reduction in collagen deposition was truly a reflection of fewer infiltrating $\mathrm{CD} 45^{+} \mathrm{Col} \mathrm{I}{ }^{+} \mathrm{CXCR} 4{ }^{+}$fibrocytes, rather than as a result of generalized migratory inhibition of other circulating CXCR4 $4^{+} \mathrm{PBMCs}$, we also examined changes in the recruitment of a panel of CXCR4 ${ }^{+}$cells to bleomycin-exposed lungs treated with anti-CXCL12 Ab's (Figure 4C). Thus, mice were treated with bleomycin and given daily injections of either antiCXCL12 or control Ab's for 8 days. Thereafter, cells were isolated from bleomycin-exposed lungs treated with either anti-CXCL12 or control Ab's and stained with a panel of markers (CD3, CD4, CD8, NK1.1, Ly6, and Mac) prior to analysis by flow cytometry. Our results show that there was no statistical difference in the numbers of $\mathrm{CD}^{+}(\mathrm{CD} 4)$ and $\mathrm{CD}^{+}(\mathrm{CD} 8) \mathrm{T}$ cells, NK cells (NK1.1), 

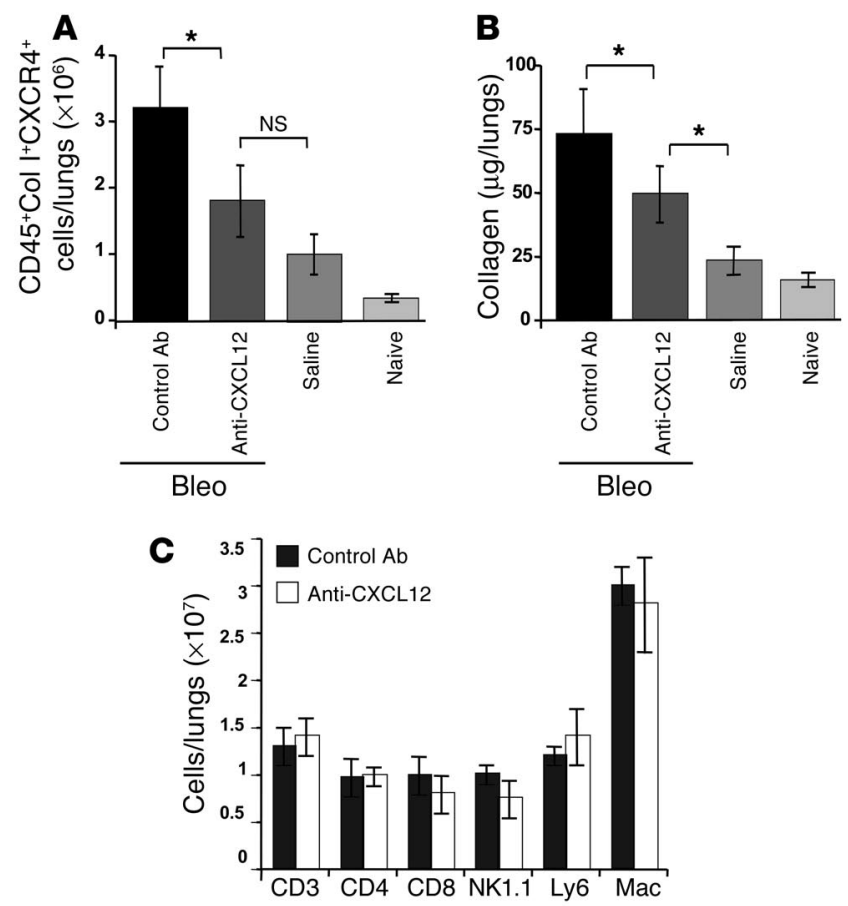

Figure 4

Neutralizing anti-CXCL12 Ab's inhibit intrapulmonary recruitment of $\mathrm{CD} 45^{+} \mathrm{Col} \mathrm{I}^{+} \mathrm{CXCR} 4{ }^{+}$fibrocytes and significantly attenuate lung fibrosis in bleomycin-treated mice. (A) Single-cell suspensions were isolated from the lungs of naive or saline- or bleomycin-exposed mice, where the bleomycin-treated mice also received daily injections of either antiCXCL12 Ab or control Ab for 16 days. These cells were then triplestained for CD45, Col I, and CXCR4 and analyzed by flow cytometry. $n=5$ samples per group. Data represent the mean \pm SEM. ${ }^{*} P<0.05$. (B) Total collagen present in lung tissue of naive, saline-, or bleomycinexposed mice, where the bleomycin-treated mice also received daily injections of either anti-CXCL12 or control Ab's for 16 days. Total collagen levels were determined by the Sircol assay. $n=4$ lungs in each group. Data represent the mean \pm SEM. ${ }^{*} P<0.05$. (C) Single-cell suspensions were isolated from the lungs of bleomycin-exposed mice treated with either daily injections of anti-CXCL12 or control Ab's for 8 days. These cells were then individually stained for CD3, CD4, CD8, NK1.1, Ly6, and Mac519 (Mac) and analyzed by flow cytometry. $n=3$ lungs per group.

neutrophils (Ly6), and monocytes/macrophages (Mac) trafficking to the lungs of bleomycin-exposed mice treated with either antiCXCL12 or control Ab's (Figure 4C). Although CXCL12 clearly does mediate recruitment of CXCR4 ${ }^{+}$cells to the lungs, the fact that neutralizing anti-CXCL12 Ab's did not block migration here can be explained by the observation that these PBMCs express chemokine receptors other than CXCR4 $(17,18)$. It is possible, therefore, that inhibition of a single chemokine receptor/ligand combination does not prevent these cell types from intrapulmonary infiltration in response to chemokines other than CXCL12.

To further verify that systemic addition of anti-CXCL12 Ab's selectively reduced intrapulmonary infiltration of $\mathrm{CD} 45^{+} \mathrm{Col} \mathrm{I}{ }^{+} \mathrm{CXCR} 4^{+}$ fibrocytes and attenuated bleomycin-induced pulmonary fibrosis, we performed histopathology on H\&E-stained lung sections (Figure $5 \mathrm{~A})$. In addition, we used the collagen-specific dye picrosirius red to stain lung sections and perform morphometric analysis (Figure 5B). For the purposes of quantitation, fibrosis was defined as areas that had an Ashcroft grade of 7 or 8 (19). These grades represent severe distortion of structure and large fibrous areas, including honeycomb lung or total fibrous obliteration of the field. By both H\&E staining (Figure 5A) and morphometric analysis (Figure 5B), anti-CXCL12 Ab's significantly reduced bleomycin-induced pulmonary fibrosis.

In addition, we immunostained lung sections exposed to bleomycin for 16 days in the presence of daily injections of either control Ab's (Figure 5C, left panel) or neutralizing CXCL12 Ab's (Figure 5C, right panel) with specific anti- $\alpha$-SMA Ab's. Levels of $\alpha$-SMA staining in those mice receiving neutralizing anti-CXCL12 was significantly lower than those mice receiving control Ab's (Figure 5C). Thus, by blocking the recruitment of CD $45^{+} \mathrm{Col} \mathrm{I}{ }^{+} \mathrm{CXCR} 4^{+}$ fibrocytes to bleomycin-exposed lungs, expression of $\alpha$-SMA was also reduced. This suggests that $\mathrm{CD} 45^{+} \mathrm{Col} \mathrm{I}{ }^{+} \mathrm{CXCR} 4^{+}$fibrocytes recruited from the peripheral circulation may ultimately develop an $\alpha$-SMA ${ }^{+}$phenotype in fibrotic lungs, which is compatible with our in vitro findings for human fibrocytes (Figure 1A and Table 1).

\section{Discussion}

This study provides, to our knowledge, the first evidence to show that circulating $\mathrm{CD} 45^{+} \mathrm{Col} \mathrm{I}{ }^{+} \mathrm{CXCR} 4^{+}$fibrocytes contribute to the pathogenesis of pulmonary fibrosis. Initially, we identified circulating pools of both human and murine $\mathrm{CD} 45^{+} \mathrm{Col} \mathrm{I}{ }^{+} \mathrm{CXCR} 4^{+}$ fibrocytes, which traffic to the lungs during pulmonary fibrosis. In the murine model of bleomycin-induced pulmonary fibrosis, exuberant collagen deposition, a key component of IPF, is observed between 4 and 20 days after bleomycin exposure. Day 8 also represents the peak period of $\mathrm{CD} 45^{+} \mathrm{Col} \mathrm{I}{ }^{+} \mathrm{CXCR} 44^{+}$fibrocyte infiltration. A CXCL12 gradient exists between the lungs and plasma of bleomycin-treated mice, which we inhibited by the addition of specific neutralizing anti-CXCL12 Ab's. Under these conditions, the trafficking of $\mathrm{CD}_{4} 5^{+} \mathrm{Col} \mathrm{I}{ }^{+} \mathrm{CXCR} 4^{+}$fibrocytes into the lungs was reduced and pulmonary fibrosis attenuated.

CXCR4 is one of about 20 chemokine receptors that have been cloned so far $(17,20-22)$, while more than 40 chemokines have also been described $(17,20-22)$. CXCL12 is the only chemokine known to bind to CXCR4, and the interplay between this receptor/ligand pair enables cellular migration to be exquisitely regulated. Although earlier studies provided conflicting results regarding the ability of $\mathrm{CXCR}^{+}$fibrocytes to migrate in response to CXCL12 in vitro, this may be explained by the fact that different methodologies were performed (6). In our assay, we counted fibrocytes that had migrated across the membrane and were adhered to the underside, while in the study of Abe and colleagues (6), only those cells that had fallen through and collected in the lower chamber were deemed to have migrated. Furthermore, we performed our chemotaxis assays in media containing $10 \%$ FCS, rather than $1 \%$ BSA, and possibly the most important factor is that we incubated our cells for 6 hours in the presence of CXCL12 rather than the 3 hours used by Abe et al. (6). In addition, recent evidence has indicated that in vitro bone marrow-derived lung $\mathrm{CXCR}^{+}{ }^{+}$cells can migrate in response to CXCL12 (23). Moreover, our in vivo studies strongly support the notion that the systemic addition of specific neutralizing anti-CXCL12 Ab's reduce intrapulmonary recruitment of $\mathrm{CD} 45^{+} \mathrm{Col} \mathrm{I}{ }^{+} \mathrm{CXCR} 4^{+}$fibrocytes and significantly attenuate lung fibrosis.

Lung fibrosis is not completely abolished by treatment with anti-CXCL12 Ab's, however. Thus, it is possible that other mechanisms could be involved in the intrapulmonary recruitment of fibrocytes and the associated development of lung fibrosis. In this respect, CCR7 is another chemokine receptor that has been 


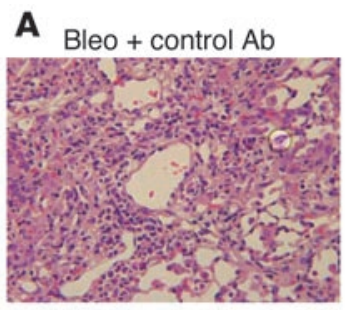

Bleo + anti-CXCL12

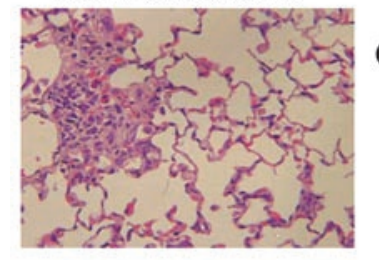

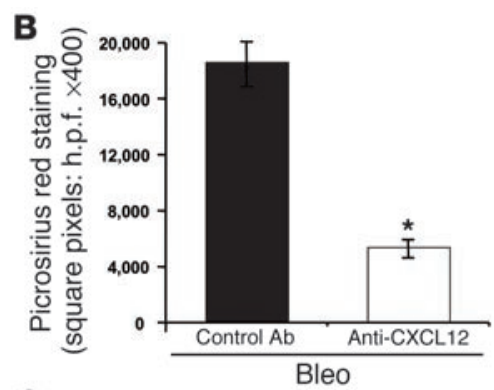

C

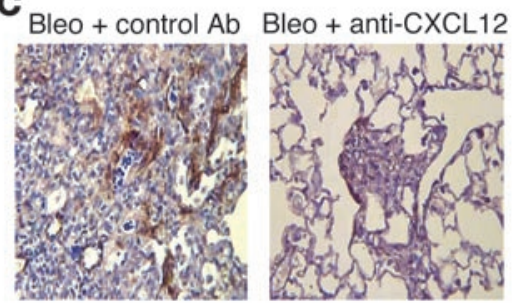

\section{Figure 5}

Representative histopathology and morphometric analysis of picrosirius red in bleomycin-induced pulmonary fibrosis and expression of $\alpha$-SMA in the presence of neutralizing anti-CXCL12 or control Ab's. (A) Representative H\&E-stained histopathologic sections of lung tissue on day 16 after intratracheal bleomycin administration in the presence of daily injections of either control (upper panel) or neutralizing anti-CXCL12 Ab's (lower panel). (B) Morphometric analysis of picrosirius red-stained lung tissue was measured by image analysis (NIH Image 1.55) and expressed as area (square pixels) at $\times 400$ magnification. ${ }^{\star} P<0.001$. (C) Histopathologic sections of lung tissue on day 16 after intratracheal bleomycin administration in the presence of daily injections of either control (left panel) or neutralizing anti-CXCL12 Ab's (right panel) stained with $\alpha$-SMA. Representative fields viewed at $\times 400$ magnification. shown to be important in the regulated movement of fibrocytes (6). Indeed, flow-cytometric analysis of our purified human fibrocytes indicated that at least two phenotypically distinct fibrocyte populations $\left(\mathrm{CD} 45^{+} \mathrm{Col} \mathrm{I}{ }^{+} \mathrm{CXCR} 4^{+}\right.$and $\left.\mathrm{CD} 45^{+} \mathrm{Col} \mathrm{I}{ }^{+} \mathrm{CXCR} 4^{-}\right)$were present. Thus it is possible that $\mathrm{CD} 45^{+} \mathrm{Col} \mathrm{I}^{+} \mathrm{CXCR} 4^{-}$fibrocytes actually express CCR7 and respond to its cognate ligands CCL19 and CCL21. To that end, we have identified a second population of fibrocytes in our mouse model that expresses CD45, Col I, and CCR7, and that also traffics to the lungs of bleomycin-exposed animals. Furthermore, it is possible that our neutralizing CXCL12 $\mathrm{Ab}$ was not completely effective in perturbing the putative CXCL12 chemotactic gradient that we believe develops between the lungs and blood plasma during bleomycin exposure and that recruits $\mathrm{CD} 45^{+} \mathrm{Col} \mathrm{I}{ }^{+} \mathrm{CXCR} 4^{+}$fibrocytes to the lungs during the onset of pulmonary fibrosis. Thus, in future studies we hope to design appropriate strategies to more fully attenuate CXCR4 and CCR7 biology and subsequently to determine whether this has an additive effect on abrogating the fibrotic response in our mouse model of bleomycin-induced pulmonary fibrosis.

In addition, several different fibroblast phenotypes, including Thy $-1^{-}$, Thy $-1^{+}$, cyclooxygenase ${ }^{\text {lo }}$, and telomerase ${ }^{+}$, have been shown to be present at the site of evolving pulmonary fibrosis $(10,24-30)$. Although it would appear that these fibroblast phenotypes may contribute to the deposition of ECM components during lung fibrosis, it is not clear whether the origin of Thy-1-, Thy $-1^{+}$, cyclooxygenase $^{\text {lo }}$, and telomerase ${ }^{+}$fibroblasts is from an intrapulmonary or extrapulmonary cellular source. If these fibroblast phenotypes originate from circulating CXCR4+ fibrocytes, it would be expected that neutralizing CXCL12 Ab's would block both the recruitment of Thy-1-, Thy- $1^{+}$, cyclooxygenase ${ }^{\text {lo }}$, and telomerase $e^{+}$fibroblasts and attenuate their ability to secrete ECM components. Alternatively, if Thy $-1^{-}$, Thy $-1^{+}$, cyclooxygenase ${ }^{\text {lo }}$, and telomerase $e^{+}$fibroblasts originate from a cellular source other than circulating CXCR4 $4^{+}$fibrocytes, then their role in mediating pulmonary fibrosis would not be impaired under conditions of blocking the CXCL12/CXCR4 biological axis. Thus, collagen deposition mediated by these additional fibroblast phenotypes could further explain why the reduction in collagen expression observed in bleomycin-exposed mice treated with anti-CXCL12 Ab's failed to reach the levels of the saline or naive control. Other cell types, including pericytes, have also been postulated to play a role in the onset of fibrosis in the liver and dermis (31-33), although there is currently no evidence to suggest that these cells are an important source of collagen deposition in pulmonary fibrosis.

A key marker of IPF is the dramatic rise in both collagen gene expression and deposition of collagen protein within the lungs. In our murine model of bleomycin-induced pulmonary fibrosis, increased expression of both pro-Col I and pro-Col III was observed beginning 4 days after instillation of bleomycin. In addition, collagen deposition was clearly detectable in lungs within 4 days of bleomycin exposure. Morphometric analysis of picrosirius red staining of lung sections at day 16 after bleomycin exposure further verified the presence of pulmonary collagen deposition. This collagen deposition was strongly attenuated in bleomycinexposed mice treated with daily injections of neutralizing antiCXCL12 Ab's. The magnitude of collagen reduction we observed here was similar to that observed in several other cytokine neutralization studies (34-39).

We measured total collagen deposition using the Sircol assay, which we felt was actually more reproducible and reliable than the hydroxyproline assay (our unpublished observations). The kinetics of collagen deposition we report here are in keeping with results found in other published work, where significant collagen deposition was detectable within 3 days of bleomycin treatment using the hydroxyproline assay (34). In addition, the concept that collagen is detectable very early in the inflammatory process is consistent with several studies that have shown markedly elevated levels of collagen during acute lung injury in patients (40-42). Indeed, increased collagen levels in the bronchoalveolar lavage (BAL) fluid of patients suffering from acute respiratory distress syndrome were detectable 3 days after the onset of the disease (40). Moreover, early detection of collagen in BAL fluid in these patients was strongly associated with an increased risk of mortality (40). This does not mean, however, that we will ultimately see fibrosis in these patients, especially in light of the magnitude of counterbalancing measures such as proteinases present at the same time, which would lead to collagen degradation, remodeling of collagen deposition, and resolution. This concept would be analogous to the saline control-treated lungs. In contrast, under conditions of bleomycin-induced pulmonary fibrosis, we speculate that the previously published find- 
ings of elevated TGF- $\beta$ in this model contributes to tipping the balance in favor of increased collagen production and at the same time decreased collagen degradation, which would result in more fibrosis. Therefore, although elevated numbers of fibrocytes may be present under conditions of saline exposure, as compared with naive mice there is no marked accumulation of collagen.

We observed significant recruitment of $\mathrm{CD} 45^{+} \mathrm{Col} \mathrm{I} \mathrm{I}^{+} \mathrm{CXCR} 4^{+}$ fibrocytes into the fibrotic lung as early as 2 days after bleomycin exposure and 2 days before significant collagen deposition was detected, further suggesting that $\mathrm{CD} 45^{+} \mathrm{Col} \mathrm{I}{ }^{+} \mathrm{CXCR} 4^{+}$fibrocytes may be involved in the onset of fibrosis. Maximal recruitment of $\mathrm{CD} 45^{+} \mathrm{Col} \mathrm{I}{ }^{+} \mathrm{CXCR} 4{ }^{+}$fibrocytes was observed 8 days after the instillation of bleomycin, and by days 16 and 20 the number of infiltrating $\mathrm{CD}_{4}{ }^{+} \mathrm{Col} \mathrm{I}{ }^{+} \mathrm{CXCR} 4^{+}$fibrocytes appeared to decrease. The fate of these cells remains to be determined, however. Indeed, the apparent loss of $\mathrm{CD} 45^{+} \mathrm{Col} \mathrm{I}{ }^{+} \mathrm{CXCR} 4^{+}$fibrocytes from fibrotic lungs, may, in fact, reflect a change in the phenotype of these cells. Myofibroblasts $\left(\mathrm{CD}^{-} 5^{-} \mathrm{Col} \mathrm{I}{ }^{+} \alpha-\mathrm{SMA}^{+}\right.$), which have been found in pulmonary fibrotic lesions but not in normal lungs (9), is one potential differentiation phenotype for fibrocytes. Although it has been suggested that myofibroblasts arise from tissue fibroblasts within the lung itself $(30,43)$, the true origin of these cells has yet to be definitively established (9). In addition, myofibroblasts are known to be a major source of pro-Col I expression in pulmonary fibrotic lesions $(9,44)$, and we have clearly demonstrated that in the presence of anti-CXCL12 Ab's collagen deposition is significantly reduced in bleomycin-exposed mice. Further support for the notion that circulating fibrocytes may ultimately differentiate into myofibroblasts within the lungs stems from our observations and other recent reports $(6,11)$ that cultured human fibrocytes eventually lose expression of CD34 and CD45 and upregulate $\alpha$-SMA, which is consistent with the known myofibroblast phenotype (CD34-CD45- $\left.\mathrm{Col} \mathrm{I}^{+} \alpha-\mathrm{SMA}^{+}\right)$(9).

Another factor that may determine the fate of $\mathrm{CD}_{4} 5^{+} \mathrm{Col} \mathrm{I}{ }^{+}$ $\mathrm{CXCR}^{+}$fibrocytes during pulmonary fibrosis is apoptosis. The inflammatory environment associated with the onset of fibrosis typically contributes extensively to the programmed cell death of injured cells, including those of the respiratory epithelium and infiltrating white blood cells. Thus, it is important for cells migrating into the lungs to have in place a mechanism to survive the apoptotic landscape. In this respect, CXCR4 ${ }^{+}$fibrocytes may be ideally situated to avoid programmed cell death since ligation of CXCR 4 by CXCL12 has been shown to regulate the cell death machinery through downstream activation of the PI3K, AKT, and MAP kinase pathways (45-49).

In conclusion, we have shown, we believe for the first time, that $\mathrm{CD}_{4} 5^{+} \mathrm{Col} \mathrm{I}{ }^{+} \mathrm{CXCR} 4^{+}$circulating fibrocytes contribute to the pathogenesis of pulmonary fibrosis. These cells, therefore, offer a novel therapeutic target for developing more effective treatments against this devastating disease.

\section{Methods}

Animal model of pulmonary fibrosis. Female C57Bl/6 mice (6-8 weeks old) purchased from The Jackson Laboratory (Bar Harbor, Maine, USA) were anesthetized with ketamine and given intratracheal injections of either $0.025 \mathrm{U}$ of bleomycin ( $0.15 \mathrm{U} / \mathrm{kg}$ Blenoxane; Sigma-Aldrich, St. Louis, Missouri, USA) or sterile saline. Mice were sacrificed for analysis 1, 2, 4, 8, 16, and 20 days after exposure to bleomycin. In separate experiments, mice treated with bleomycin were given daily injections $(500 \mu \mathrm{l})$ of $10 \mathrm{mg} / \mathrm{ml}$ $\mathrm{F}\left(\mathrm{ab}^{\prime}\right)_{2}$ fragments of either neutralizing goat anti-CXCL12 $\mathrm{Ab}$ or control
Ab's for 8 or 16 days prior to sacrifice. Animal use for these studies was approved by the Department of Laboratory Animal Medicine, David Geffen School of Medicine at UCLA.

Lung tissue preparation for protein assays. Bleomycin- or saline control-treated lungs were homogenized and sonicated in complete buffer (Roche Diagnostics Corp., Indianapolis, Indiana, USA) as previously described (41). Homogenates were centrifuged at $900 \mathrm{~g}$ for 15 minutes, filtered through $1.2 \mu \mathrm{m}$ Sterile Acrodiscs (Gelman Sciences Inc., Ann Arbor, Michigan, USA), and frozen at $-70^{\circ} \mathrm{C}$ until required.

CXCL12 ELISA. Lung homogenate and plasma were assayed for CXCL12 levels as previously described (50).

Sircol assay. The Sircol collagen assay (Biocolor Ltd., Belfast, United Kingdom) was performed following the manufacturer's instructions (13). Briefly, Sirius red reagent $(50 \mu \mathrm{l})$ was added to each lung homogenate (50 $\mu \mathrm{l})$ and mixed for 30 minutes. The collagen-dye complex was precipitated by centrifugation at $16,000 \mathrm{~g}$ for 5 minutes, washed with ethanol, and dissolved in $0.5 \mathrm{M} \mathrm{NaOH}$. Finally, the samples were introduced into a microplate reader and the absorbance determined at $540 \mathrm{~nm}$.

Total RNA isolation and real-time quantitative RT-PCR. Total RNA was prepared using TRIzol and by following the manufacturer's instructions. Real-time quantitative RT-PCR was performed using specific TaqMan primers and probes, the ABI Prism 7700 sequence detector, and SDS analysis software (Applied Biosystems, Foster City, California, USA), as previously described (50).

Purification of circulating human fibrocytes. Human fibrocytes were isolated according to previously published methods $(5,6,11)$. Briefly, PBMCs were isolated from leukopheresis packs (HemaCare Inc., Woodland Hills, California, USA) using Ficoll density centrifugation. Cells isolated in this way were then cultured in DMEM supplemented with $20 \%$ FCS, penicillin, streptomycin, and L-glutamine for 7-14 days. Typically, $2 \times 10^{7} \mathrm{PBMCs}$ were plated into each T150 tissue culture flask. After 7-14 days, the media was aspirated from the PBMC culture and the remaining adherent cells washed with sterile PBS. Subsequently, the adherent cells were harvested using Accutase (Innovative Cell Technologies Inc., San Diego, California, USA), which we have found to be more efficient than the standard trypsin protocol for removing adherent cells from plastic. Previous data (6) have indicated that the main contaminating cell types in these cultures are B lymphocytes (about 3\%), T lymphocytes (about 13\%), and monocytes/macrophages (up to $20 \%$ of the PBMC culture). Thus, we used immunomagnetic selection to deplete B lymphocytes (Dynabeads M-450 anti-CD19; Dynal Inc., Lake Success, New York, USA), T lymphocytes (Dynabeads M-450 anti-CD2), and monocytes/macrophages (Dynabeads M-450 anti-CD14) from our crude fibrocyte preparation. Purified fibrocytes prepared in this way were returned to culture (DMEM supplemented with 20\% FCS, penicillin, streptomycin and L-glutamine) for an additional 5 days before analysis. Fibrocytes were routinely greater than $90 \%$ pure as determined by staining with CD $45, \mathrm{Col}$ I, and CXCR4. Typically, our isolated fibrocytes represented about $0.5 \%$ of the overall pool of peripheral white blood cells.

FACS analysis. Cells isolated from the bone marrow, lungs, and peripheral circulation of mice were initially stained with FITC-labeled CXCR4 or FITClabeled CCR7 and peridinin chlorophyll- $a$ protein-labeled (PerCP-labeled) CD45. Subsequently, the cells were permeabilized using cytofix/cytoperm (BD Biosciences - Pharmingen, San Diego, California, USA) to facilitate intracellular staining of Col I. Col I was stained initially with anti-human or anti-mouse Col I (Chemicon International, Temecula, California, USA) and then phycoerythrin-conjugated (PE-conjugated) goat anti-rabbit Ig (Molecular Probes Inc., Eugene, Oregon, USA). Three-color analysis of the stained cells was performed on a FACScan flow cytometer (BD Biosciences - Pharmingen) using Cellquest 3.2.1f1 software. In separate experiments cells isolated from the lungs were individually stained with PE-conjugated 
CD3, CD4, CD8, NK1.1, LY.6, and Mac519 (Serotec Inc., Raleigh, North Carolina, USA). Except where indicated, all $\mathrm{mAb}$ 's were purchased from $\mathrm{BD}$ Biosciences - Pharmingen. To obtain sufficient cells from the blood for analysis by FACS, two buffy coats ( $500 \mu \mathrm{l}$ per buffy coat) were combined from each treatment condition. Thus data from fibrocytes isolated from the peripheral circulation are presented as cells per milliliter of blood.

Purified human fibrocytes cultured for either 1, 2, or 3 weeks after their initial isolation were taken and stained with FITC-labeled CXCR4, APC-labeled CD34, and PerCP-labeled CD45. Next, the cells were permeabilized using cytofix/cytoperm to facilitate intracellular staining of Col I and $\alpha$-SMA. Col I and $\alpha$-SMA were then stained with unconjugated rabbit anti-human Col I and mouse anti-human $\alpha$-SMA Ab's followed by anti-rabbit FITC and anti-mouse FITC, respectively. Finally, single-color analysis of the stained cells was performed on a FACScan flow cytometer using Cellquest 3.2.1f1 software.

Preparation of $F\left(a b^{\prime}\right)_{2}$ fragments of goat anti-human CXCL12 whole Ig. $\mathrm{F}\left(\mathrm{ab}^{\prime}\right)_{2}$ fragments of goat anti-human CXCL12 (16) were prepared using the ImmunoPure $\mathrm{F}\left(\mathrm{ab}^{\prime}\right)_{2}$ kit (Pierce Chemical Co., Rockford, Illinois, USA). Briefly, immobilized pepsin was used to digest whole Ig into $\mathrm{Fc}$ and $\mathrm{F}\left(\mathrm{ab}^{\prime}\right)_{2}$ fractions. These fractions were then separated by protein A-affinity chromatography such that the Fc portion bound to the column, while the $\mathrm{F}\left(\mathrm{ab}^{\prime}\right)_{2}$ portion was eluted, collected, and further purified by dialyzing against PBS, pH 7.4, overnight.

Fibrocyte chemotaxis. Chemotaxis was performed using fibronectin-coated filters ( $5 \mu \mathrm{m}$ pore) as previously described (16).

Histology. Lungs were perfused with saline and then inflated with $1 \mathrm{ml}$ of $4 \%$ paraformaldehyde at a constant pressure of $30 \mathrm{cmH}_{2} \mathrm{O}$. Lungs were ligated at the trachea, removed en bloc, and immersed in $4 \%$ paraformaldehyde for 24 hours, at which time they were changed to $70 \%$ alcohol before paraffin embedding, followed by sectioning and $\mathrm{H} \& \mathrm{E}$ staining.

Morphometric analysis and picrosirius red staining. Paraffin-embedded sections ( $3 \mu \mathrm{m}$ thick) of bleomycin-exposed lungs treated with either a control $\mathrm{Ab}$ or neutralizing anti-CXCL12 Ab were exposed to xylene (twice, 1 minute each) and then washed initially in $100 \%$ ethanol (twice, 1 minute), followed by $50 \%$ ethanol (twice, 1 minute), and finally rinsed in distilled water for 10 minutes. Subsequently, the sections were treated with $0.2 \%$ phosphomolybdic acid ( 2 minutes), rinsed in distilled water ( 2 minutes), and then exposed to a $0.1 \%$ solution of picrosirius red stain for 90 minutes. After picrosirius red staining, the sections were again rinsed in distilled water ( 1 minute) and then $50 \%$ ethanol (twice, 1 minute each), 100\% ethanol (twice, 1 minute each), and finally xylene ( 1 minute). Next, ten fields per section ( $n=8$ sections per lung) of random lung specimens ( $n=5$ lungs per group) were morphometrically assessed for the magnitude of picrosirius red staining. To quantitate the areas of positive picrosirius red staining, we used NIH 1.55 imaging software (37).
Immunostaining. Histopathologic sections of lung tissue were prepared from mice who had been exposed to bleomycin for 16 days in the presence of either control or neutralizing anti-CXCL12 Ab's and assessed by immunostaining of $\alpha$-SMA (R\&D Systems Inc., Minneapolis, Minnesota, USA). Purified fibrocytes ( $10^{4}$ cells per well) were cultured on TissueTechs (BD Biosciences - Pharmingen) for the times indicated and then immunostained. Briefly, the cells were fixed in $4 \%$ paraformaldehyde and then incubated with a $1: 1$ mixture of $3 \%$ hydrogen peroxide in methanol. Subsequently, the cells were exposed to Power Block (BioGenex Laboratories, San Ramon, California, USA) for 30 minutes and, after washing in $\mathrm{PBS}$, stained with a primary $\mathrm{Ab}$ (human and murine Col I from Chemicon International; CXCR4 from R\&D Systems Inc.; $\alpha$-SMA; or an isotype control) for a further 30 minutes at room temperature. After incubation with the primary Ab, the cells were washed with PBS and then exposed to a biotinylated anti-mouse secondary $\mathrm{Ab}$ for 30 minutes. Subsequently, the cells were washed in PBS and avidin-binding complex (Vector Laboratories, Burlingame, California, USA). Reagent was added, and the cells incubated again for 30 minutes. After an additional PBS wash, the cells were exposed to the chromogen 3,3'-diaminobenzidine tetrahydrochloride (Vector Laboratories), which turns the positively staining cells brown. Finally, the cells were counterstained with hematoxylin, neutralized in $10 \%$ ammonia, and left to dry overnight. After drying overnight, Permount was added, together with a coverslip, and the cells were then viewed by microscopy.

Statistical analysis. The animal studies involved 3-6 mice for each treatment group. Data were analyzed on a Dell PC (Dell, Round Rock, Texas, USA) computer using the Statview 5.0 statistical package (Abacus Concepts Inc., Berkeley, California, USA). Comparisons were evaluated by Student's unpaired $t$ test. Results were statistically significant if $P$ values were 0.05 or less.

\section{Acknowledgments}

Funding was provided by NIH grants HL-66027 (to R.M. Strieter), P50HL-67665 (to M.P. Keane and R.M. Strieter), and HL-03906 (to M.P. Keane).

Received for publication January 7, 2004, and accepted in revised form June 15, 2004.

Address correspondence to: Robert M. Strieter, Division of Pulmonary and Critical Care Medicine, Departments of Medicine, Pathology, and Laboratory Medicine, University of California - Los Angeles, School of Medicine, 900 Veteran Avenue, 14-154 Warren Hall, Los Angeles, California 90095-1786, USA. Phone: (310) 794-1999; Fax: (310) 794-1998; E-mail: rstrieter@mednet.ucla.edu.
1. Coultas, D.B., Zumwalt, R.E., Black, W.C., and Sobonya, R.E. 1994. The epidemiology of interstitial lung diseases. Am. J. Respir. Crit. Care Med. 150:967-972.

2. Perez, A., Rogers, R.M., and Dauber, J.H. 2003. The prognosis of idiopathic pulmonary fibrosis. Am. J. Respir. Cell Mol. Biol. 29(3 Suppl.):S19-S26.

3. du Bois, R. 1997. Diffuse lung disease: a view for the future. Sarcoidosis Vasc. Diffuse Lung Dis. 14:23-30.

4. Dacic, S., and Yousem, S.A. 2003. Histologic classification of idiopathic chronic interstitial pneumonias. Am. J. Respir. Cell Mol. Biol. 29(3 Suppl.):S5-S9.

5. Bucala, R., Spiegel, L.A., Chesney, J., Hogan, M., and Cerami, A. 1994. Circulating fibrocytes define a new leukocyte subpopulation that mediates tissue repair. Mol. Med. 1:71-81.

6. Abe, R., Donnelly, S.C., Peng, T., Bucala, R., and Metz, C.N. 2001. Peripheral blood fibrocytes: differentiation pathway and migration to wound sites. J. Immunol. 166:7556-7562.
7. Metz, C.N. 2003. Fibrocytes: a unique cell population implicated in wound healing. Cell. Mol. Life Sci. 60:1342-1350.

8. Chesney, J., Metz, C., Stavitsky, A.B., Bacher, M., and Bucala, R. 1998. Regulated production of type I collagen and inflammatory cytokines by peripheral blood fibrocytes. J. Immunol. 160:419-425.

9. Phan, S.H. 2003. Fibroblast phenotypes in pulmonary fibrosis. Am. J. Respir. Cell Mol. Biol. 29(3 Suppl.):S87-S92.

10. Fries, K.M., et al. 1994. Evidence of fibroblast heterogeneity and the role of fibroblast subpopulations in fibrosis. Clin. Immunol. Immunopathol. 72:283-292.

11. Schmidt, M., Sun, G., Stacey, M.A., Mori, L., and Mattoli, S. 2003. Identification of circulating fibrocytes as precursors of bronchial myofibroblasts in asthma. J. Immunol. 171:380-389.

12. Chandler, D.B. 1990. Possible mechanisms of bleomycin-induced fibrosis. Clin. Chest Med. 11:21-30.
13. Blease, K., et al. 2002. Stat6-deficient mice develop airway hyperresponsiveness and peribronchial fibrosis during chronic fungal asthma. Am.J. Pathol. 160:481-490.

14. Epperly, M.W., Guo, H., Gretton, J.E., and Greenberger, J.S. 2003. Bone marrow origin of myofibroblasts in irradiation pulmonary fibrosis. Am. J. Respir. Cell Mol. Biol. 29:213-224.

15. Muller, A., et al. 2001. Involvement of chemokine receptors in breast cancer metastasis. Nature. 410:50-56.

16. Phillips, R.J., et al. 2003. The stromal derived factor-1/CXCL12-CXC chemokine receptor 4 biological axis in non-small cell lung cancer metastases. Am. J. Respir. Crit. Care Med. 167:1676-1686.

17. Baggiolini, M. 1998. Chemokines and leukocyte traffic. Nature. 392:565-568.

18. Homey, B., Muller, A., and Zlotnik, A. 2002. Chemokines: agents for the immunotherapy of cancer? Nat. Rev. Immunol. 2:175-184. 
19. Ashcroft, T., Simpson, J.M., and Timbrell, V. 1988 Simple method of estimating severity of pulmonary fibrosis on a numerical scale. J. Clin. Pathol. 41:467-470.

20. Murphy, P.M., et al. 2000. International union of pharmacology. XXII. Nomenclature for chemokine receptors. Pharmacol. Rev. 52:145-176.

21. Murphy, P.M. 2002. International Union of Pharmacology. XXX. Update on chemokine receptor nomenclature. Pharmacol. Rev. 54:227-229.

22. Premack, B.A., and Schall, T.J. 1996. Chemokine receptors: gateways to inflammation and infection. Nat. Med. 2:1174-1178.

23. Hashimoto, N., Jin, H., Liu, T., Chensue, S.W., and Phan, S.H. 2004. Bone marrow-derived progenitor cells in pulmonary fibrosis. J. Clin. Invest. 113:243-252. doi:10.1172/JCI200418847.

24. Derdak, S., et al. 1992. Differential collagen and fibronectin production by Thy $1+$ and Thy $1-$ lung fibroblast subpopulations. Am. J. Physiol. 263:L283-L290.

25. McIntosh, J.C., Hagood, J.S., Richardson, T.L., and Simecka, J.W. 1994. Thy1 (+) and (-) lung fibrosis subpopulations in LEW and F344 rats. Eur. Respir. J. 7:2131-2138

26. Silvera, M.R., Sempowski, G.D., and Phipps, R.P. 1994. Expression of TGF-beta isoforms by Thy-1+ and Thy-1- pulmonary fibroblast subsets: evidence for TGF-beta as a regulator of IL-1-dependent stimulation of IL-6. Lymphokine Cytokine Res. 13:277-285.

27. Wilborn, J., et al. 1995. Cultured lung fibroblasts isolated from patients with idiopathic pulmonary fibrosis have a diminished capacity to synthesize prostaglandin E2 and to express cyclooxygenase-2. J. Clin. Invest. 95:1861-1868.

28. Nozaki, Y., Liu, T., Hatano, K., Gharaee-Kermani, M., and Phan, S.H. 2000. Induction of telomerase activity in fibroblasts from bleomycin-injured lungs. Am. J. Respir. Cell Mol. Biol. 23:460-465.

29. Liu, T., Nozaki, Y., and Phan, S.H. 2002. Regulation of telomerase activity in rat lung fibroblasts. Am.J. Respir. Cell Mol. Biol. 26:534-540.
30. Hashimoto, S., Gon, Y., Takeshita, I., Maruoka, S., and Horie, T. 2001. IL-4 and IL-13 induce myofibroblastic phenotype of human lung fibroblasts through c-Jun NH2-terminal kinase-dependent pathway. J. Allergy Clin. Immunol. 107:1001-1008.

31. Niki, T., et al. 1999. Class VI intermediate filament protein nestin is induced during activation of rat hepatic stellate cells. Hepatology. 29:520-527.

32. Sundberg, C., Ivarsson, M., Gerdin, B., and Rubin, K. 1996. Pericytes as collagen-producing cells in excessive dermal scarring. Lab. Invest. 74:452-466.

33. Sato, M., Suzuki, S., and Senoo, H. 2003. Hepatic stellate cells: unique characteristics in cell biology and phenotype. Cell Struct. Funct. 28:105-112.

34. Gurujeyalakshmi, G., and Giri, S.N. 1995. Molecular mechanisms of antifibrotic effect of interferon gamma in bleomycin-mouse model of lung fibrosis: downregulation of TGF-beta and procollagen I and III gene expression. Exp. Lung Res. 21:791-808.

35. Piguet, P.F., Vesin, C., Grau, G.E., and Thompson, R.C. 1993. Interleukin 1 receptor antagonist (IL-1ra) prevents or cures pulmonary fibrosis elicited in mice by bleomycin or silica. Cytokine. 5:57-61.

36. Piguet, P.F., Vesin, C., and Thomas, F. 1995. Bombesin down modulates pulmonary fibrosis elicited in mice by bleomycin. Exp. Lung Res. 21:227-237.

37. Keane, M.P., et al. 1999. IFN-gamma-inducible protein-10 attenuates bleomycin-induced pulmonary fibrosis via inhibition of angiogenesis. J. Immunol. 163:5686-5692.

38. Keane, M.P., Belperio, J.A., Burdick, M.D., and Strieter, R.M. 2001. IL-12 attenuates bleomycininduced pulmonary fibrosis. Am. J. Physiol. Lung Cell. Mol. Physiol. 281:L92-L97.

39. Keane, M.P., et al. 1999. Neutralization of the CXC chemokine, macrophage inflammatory protein-2, attenuates bleomycin-induced pulmonary fibrosis. J. Immunol. 162:5511-5518.

40. Clark, J.G., Milberg, J.A., Steinberg, K.P., and Hudson, L.D. 1995. Type III procollagen peptide in the adult respiratory distress syndrome. Association of increased peptide levels in bronchoalveolar lavage fluid with increased risk for death. Ann. Intern. Med. 122:17-23.

41. Martin, C., Papazian, L., Payan, M.J., Saux, P., and Gouin, F. 1995. Pulmonary fibrosis correlates with outcome in adult respiratory distress syndrome. A study in mechanically ventilated patients. Chest. 107:196-200.

42. Pugin, J., Verghese, G., Widmer, M.C., and Matthay, M.A. 1999. The alveolar space is the site of intense inflammatory and profibrotic reactions in the early phase of acute respiratory distress syndrome. Crit. Care Med. 27:304-312.

43. Serini, G., and Gabbiani, G. 1999. Mechanisms of myofibroblast activity and phenotypic modulation. Exp. Cell Res. 250:273-283.

44. Zhang, K., Rekhter, M.D., Gordon, D., and Phan, S.H. 1994. Myofibroblasts and their role in lung collagen gene expression during pulmonary fibrosis. A combined immunohistochemical and in situ hybridization study. Am. J. Pathol. 145:114-125.

45. Vicente-Manzanares, M., et al. 1999. Involvement of phosphatidylinositol 3-kinase in stromal cellderived factor- 1 alpha-induced lymphocyte polarization and chemotaxis. J. Immunol. 163:4001-4012.

46. Sotsios, Y., Whittaker, G.C., Westwick, J., and Ward, S.G. 1999. The CXC chemokine stromal cell-derived factor activates a Gi-coupled phosphoinositide 3kinase in T lymphocytes. J. Immunol. 163:5954-5963.

47. Suzuki, Y., Rahman, M., and Mitsuya, H. 2001. Diverse transcriptional response of CD4(+) T cells to stromal cell-derived factor (SDF)-1: cell survival promotion and priming effects of SDF-1 on CD4(+) T cells. J. Immunol. 167:3064-3073.

48. Cardone, M.H., et al. 1998. Regulation of cell death protease caspase- 9 by phosphorylation. Science. 282:1318-1321.

49. Brunet, A., et al. 1999. Akt promotes cell survival by phosphorylating and inhibiting a Forkhead transcription factor. Cell. 96:857-868.

50. Belperio, J.A., et al. 2002. Interaction of IL-13 and C10 in the pathogenesis of bleomycin-induced pulmonary fibrosis. Am. J. Respir. Cell Mol. Biol. 27:419-427. 